\title{
Obesity in older adults: effect of degree of weight loss on cardiovascular markers and medications.
}

Cilla J Haywood ${ }^{1,3,4}$

Luke A Prendergast ${ }^{6}$

Ratana $\operatorname{Lim}^{7}$

Martha Lappas ${ }^{7}$

Wen Kwang Lim ${ }^{2,5}$

Joseph Proietto ${ }^{1,4}$

1. Department of Medicine (Austin Health), University of Melbourne, Heidelberg Heights, Australia

2. Department of Medicine (Royal Melbourne Hospital), University of Melbourne, Parkville, Australia

3. Department of Aged Care, Austin Health, Heidelberg, Australia

4. Department of Endocrinology, Austin Health, Heidelberg, Australia

5. Department of Aged Care, Royal Melbourne Hospital, Parkville, Australia

6. Mathematics and Statistics, LaTrobe University, Bundoora, Australia

7. Obstetrics, Nutrition and Endocrinology Group, Department of Obstetrics and Gynaecology, University of Melbourne, Mercy Hospital for Women, Heidelberg, Victoria, Australia

Corresponding Author: Dr. Cilla Haywood

chaywood@unimelb.edu.au

Metabolic Disorders Centre

$1^{\text {st }}$ Floor, Centaur Wing, Heidelberg Repatriation Hospital

Waterdale Rd, Heidelberg Heights, Victoria 3081, Australia

$\mathrm{Ph}+61407340863$

Orcid ID for Cilla Haywood: https://orcid.org/0000-0002-8127-7014

Running title: Weight loss in older persons with obesity

Keywords: Obesity, older adults, hypertension, very low calorie diet

This is the author manuscript accepted for publication and has undergone full peer review but has not been through the copyediting, typesetting, pagination and proofreading process, which may lead to differences between this version and the Version of Record. Please cite this article as doi: $10.1111 /$ cob.12316

This article is protected by copyright. All rights reserved. 
Conflict of Interest Statement: The authors report no conflicts of interest directly related to this study. Related to broader study of obesity, $\mathrm{CH}$ and JP have received lecture fees from Novo Nordisk and iNova Pharmaceuticals (Duromine ${ }^{\circledR}$ ). JP is on the medical advisory board for Saxenda ${ }^{\circledR}$ (Novo Nordisk Pharmaceuticals)

Acknowledgements: The research project was funded a National Health and Medical Research Council Project grant (APP1044334), and CH was supported by a National Health and Medical Research Council Medical/Dental Postgraduate Scholarship (APP1056512).

\title{
Obesity in older adults: effect of degree of weight loss on cardiovascular markers and medications.
}

\section{What is already known about this subject:}

- Obesity is an increasing issue in older adults, increasing morbidity, polypharmacy and the risk of disability.

- Modest weight loss, achieved with diet and exercise, is feasible and safe in older persons with obesity.

- Intentional weight loss can improve cardiovascular markers in this group.

\section{What this study adds:}

- This study demonstrates the use of very low calorie diets (VLCDs) in older adults with obesity.

- The effect of more rapid weight loss on cardiovascular markers and cardiovascular medication usage in this group is also demonstrated.

\begin{abstract}
:
Background: Obesity worsens the age-related tendency towards cardiovascular disease and diabetes. Older adults are vulnerable to medication adverse effects. Intentional weight loss in older adults with obesity has been shown to improve cardiovascular and glycaemic markers. The effect of rapid weight loss induced by Very Low Calorie Diets (VLCDs) on these markers has not been evaluated in this group.
\end{abstract}

Methods: In this 12-week study, participants were randomized to one of healthy eating, hypocaloric diet or VLCD, all combined with three times weekly exercise (Ex/HE, Ex/Diet, 
$\mathrm{Ex} / \mathrm{VLCD}$, respectively). The effects of these interventions on weight, blood pressure, lipids, glucose and $\mathrm{HbA1c}$, inflammatory markers and cardiovascular and diabetes medication changes were measured.

Results: Weight loss was 3.7, 5.1, and 11.1\% in Ex/HE, Ex/Diet and Ex/VLCD, respectively. There were significant improvements in $\mathrm{HbA}_{1 \mathrm{c}}$ in all groups, but by the greatest degree in Ex/VLCD $(0.18 \pm 0.07,0.18 \pm 0.06$ and $0.59 \pm 0.13 \%$, respectively). Similar patterns were seen in total cholesterol $(0.13 \pm 0.15,0.21 \pm 0.11$ and $0.53 \pm 0.13 \mathrm{mmol} / \mathrm{L}$, respectively, $\mathrm{p}=0.047)$, triglycerides $(0.35 \pm 0.13,0.20 \pm 0.10$ and $0.51 \pm 0.09 \mathrm{mmol} / \mathrm{L}$, respectively, $\mathrm{p}=0.011)$ and systolic blood pressure $(9 \pm 2,2 \pm 3$ and $14 \pm 3 \mathrm{mmHg}$ respectively, $\mathrm{p}=0.025)$. There were no between-group differences in fasting glucose, high-density lipoprotein (HDL) cholesterol, LDL-C and inflammatory markers. Reductions in antihypertensive or diabetes medication were made in 4/29, 7/36 and 16/37 participants in Ex/HE, Ex/Diet and Ex/VLCD, respectively ( $p=0.017$ )

Conclusions: Significant weight loss achieved with a VLCD gave rise to improvements in multiple cardiovascular risk markers, despite reduction in medication. Weight loss is an underutilised method of cardiovascular risk management in this group.

\section{Key Words:}

Reducing diet

Very Low Calorie Diet

Obesity

Hypertension

Diabetes

This article is protected by copyright. All rights reserved. 


\section{Obesity in older adults: effect of degree of weight loss on cardiovascular markers and medications.}

Ageing and obesity can worsen cardiovascular risk factors and insulin resistance, and the inflammatory milieu seen in both conditions is implicated in the pathophysiology of diseases of ageing $^{1,2}$. Intentional weight loss and/or exercise can improve or modify these risk factors. For most of these risk factors, the degree of improvement correlates with the degree of weight loss, and are greater in lower carbohydrate diets and VLCDs than in low fat diets ${ }^{3,4}$. Exercise gives small but statistically significant improvements in cardiovascular parameters ${ }^{5}$. Improvements in pancreatic function ${ }^{6}$, blood pressure, cholesterol ${ }^{7}$ and inflammatory markers ${ }^{8}$ have also been observed in studies focussing on older people with obesity. This 12 -week study compared the effects of exercise with healthy eating advice (Ex/HE), exercise with hypocaloric diet (Ex/Diet) and exercise with VLCD (Ex/VLCD) on blood glucose, haemoglobin $\mathrm{A}_{1} \mathrm{C}\left(\mathrm{HbA}_{1 \mathrm{C}}\right)$, lipid parameters, blood pressure and inflammatory markers. We hypothesised that those on the VLCD would have the greatest improvements in blood pressure, diabetes and inflammatory markers

\section{Methods and Materials}

$\underline{\text { Study Oversight }}$

This article is protected by copyright. All rights reserved. 
We conducted this study from August 2012 until July 2015 at the Heidelberg Repatriation Hospital. The study was registered with the Australian and New Zealand Clinical Trials Registry (ACTRN12611000408987), and approved by the Austin Health Research Ethics Committee.

Details regarding the recruitment, participants, monitoring and interventions in this study have been published previously elsewhere ${ }^{9}$. Briefly, volunteers were eligible for the study if they were 65 years or over, community dwelling, and had a body mass index (BMI) e $35 \mathrm{~kg} / \mathrm{m}^{2}$. A baseline visit was undertaken, where blood pressure was taken, and blood drawn for lipids, glucose and inflammatory markers. Physical function and cognitive parameters were also tested. Participants were randomised to three-times weekly exercise with one of:

- a general healthy eating plan (Ex/HE),

- hypocaloric high protein diet (Ex/Diet), or

- $\quad$ very low calorie diet (Ex/VLCD).

Baseline measurements were repeated at 12 weeks. Adherence to dietary interventions was monitored via food charting and inferred from monitoring of weight. Attendance at exercise classes was recorded.

Blood pressure and glucose monitoring (Safety Measures)

Participants visited the study centre every two weeks, and their weight, waist circumference and blood pressure was measured. Blood pressure was measured once, sitting, after a 5-minute rest,

This article is protected by copyright. All rights reserved. 
using a mercury sphygmomanometer and an appropriately sized cuff. A physician reviewed the blood pressure and advised on reduction of antihypertensives if blood pressure was below 120/80 $\mathrm{mmHg}$, with the aim of minimizing the risk of symptomatic hypotension. Changes to antihypertensive medications were recorded.

Participants with type- 2 diabetes were asked to check their blood glucose 4 times per day, before meals. Those randomized to a VLCD who were taking insulin or sulfonylureas had the dosage of these medications halved, as previously published elsewhere ${ }^{10}$. They notified the physician if their blood glucose dropped below $5 \mathrm{mmol} / \mathrm{L}$, to change medications before frank hypoglycaemia ensued. Hypoglycaemic episodes and changes to medications were recorded.

\section{Inflammatory parameters}

Serum was frozen and stored at $-18^{0} \mathrm{C}$, and batch assayed for TNF- \pm and IL-6 levels using high sensitivity Quantikine ELISA kits (R\&D systems, Minneapolis, MN, USA). The limit of detection for both assays was $0.2 \mathrm{pg} / \mathrm{ml}$. The calculated inter-assay and intra-assay coefficients of variation $(\mathrm{CV})$ were all less than $10 \%$.

\section{Statistical analysis}

When considering change between baseline and completion, many measures exhibited skewness. Consequently, we used the Kruskal-Wallis test for comparisons across the three groups. To 
model completion levels, we conducted multiple linear regression analyses adjusting for baseline values, age and gender by including them as covariates along with the factor variable group (3 levels). Estimated models were all checked to ensure model and error conditions were valid. Models for several variables (BGL, HbA1c, LDL, TNF- \pm and IL-6) revealed violations and improvements were made following a log-transformation of the dependent variable. Pairwise comparisons between groups for the non-transformed models were carried out using Tukey adjustments for multiple comparisons and reported as differences in mean values between groups. For the log-transformed models, pairwise comparisons were first made on the log scale, before being converted to percentage difference in mean values between groups and again the Tukey method was used for multiple comparisons. Categorical variables were compared using Pearson's Chi-Square or Fisher's Exact test.

Power calculations for the study were made on the basis of the physical function tests and have been described elsewhere?

\section{Results}

Study population:

There were 117 participants, and 102 completed the study. 37 participants had diabetes and 95 had a history of hypertension (table 1). There were no significant between-group differences in baseline characteristics. The mean age was 70 years (range 65-85), mean BMI 40kg/m² (range 32.3-57) and 72 were female. Attrition was 7/36, 4/40 and 4/41 in Ex/HE, Ex/Diet and 
Ex/VLCD, respectively. Weight was reduced by $3.7,5.1$ and $11.1 \%$, respectively $(\mathrm{p}<0.001)$.

Mean baseline systolic and diastolic blood pressure was $142 \pm 15$ and $82 \pm 10 \mathrm{mmHg}$, respectively. Mean baseline fasting blood glucose and total cholesterol was $6.2 \pm 2.2$ and $4.9 \pm 1.2 \mathrm{mmol} / \mathrm{L}$, respectively. No significant between-group difference was found for any of the baseline measures.

Adverse effects:

The adverse effects of study interventions have been published ${ }^{9}$. Briefly, despite halving insulin at baseline, two participants in Ex/VLCD had hypoglycaemia (BSLs 2.9 and $3.2 \mathrm{mmol} / \mathrm{L}$ ). Both resolved with ingestion of carbohydrates and no events occurred after further reduction of insulin dose. Subjective complaints of dizziness occurred in 0/36, 1/40 participants and 3/41 participants in $\mathrm{Ex} / \mathrm{HE}, \mathrm{Ex} / \mathrm{Diet}$ and $\mathrm{Ex} / \mathrm{VLCD}$, respectively $(\mathrm{p}=0.324)$

\section{Glycaemic Parameters}

Blood glucose was reduced by $0.12 \pm 0.2,0.05 \pm 0.15$ and $0.5 \pm 0.27 \mathrm{mmol} / \mathrm{L}$ in $\mathrm{Ex} / \mathrm{HE}, \mathrm{Ex} / \mathrm{Diet}$ and $\mathrm{Ex} / \mathrm{VLCD}$, respectively $(\mathrm{p}=0.466) . \mathrm{HbA}_{1 \mathrm{c}}$ was reduced significantly from baseline in all groups, but by the greatest degree in Ex/VLCD; $0.18 \pm 0.07,0.18 \pm 0.06$ and $0.59 \pm 0.13 \%$, respectively $(\mathrm{p}=0.018)$ (Table 2). Reduction in diabetes medication was required in $2 / 9,3 / 12$ and 10/14 participants with diabetes in Ex/HE, Ex/Diet and Ex/VLCD, respectively ( $p=0.028$ ) (table 3)

\section{Lipid Parameters}

This article is protected by copyright. All rights reserved. 
Total cholesterol decreased by $0.13 \pm 0.15,0.21 \pm 0.11$ and $0.53 \pm 0.13 \mathrm{mmol} / \mathrm{L}$, respectively $(\mathrm{p}=0.047)$, and triglycerides decreased by $0.35 \pm 0.13,0.20 \pm 0.10$ and $0.51 \pm 0.09 \mathrm{mmol} / \mathrm{L}$, respectively $(\mathrm{p}=0.011)$ (Table 2$)$.

\section{Blood Pressure Parameters}

Systolic blood pressure decreased by $9 \pm 2,2 \pm 3$ and $14 \pm 3 \mathrm{mmHg}$ in Ex/HE, Ex/Diet and Ex/VLCD, respectively $(p=0.025)$. There was no significant between-group difference in change in diastolic blood pressure $(\mathrm{p}=0.909)$ (Table 2). Reduction in antihypertensive medication was required in 0/27, 5/32 and 16/37 completers in Ex/HE, Ex/Diet and Ex/VLCD, respectively $(\mathrm{p}=0.006)($ Table 3$)$.

Inflammatory Parameters

There was no significant reduction in TNF- \pm or IL-6 in any group, nor were there any betweengroup change in these parameters in $(\mathrm{p}=0.225$ and 0.728 , respectively) (Table 2$)$.

\section{Discussion}

Ageing is associated with a number of physiological changes, which predispose to cardiovascular disease. These are decreased insulin sensitivity ${ }^{11}$; increased arterial stiffness leading to hypertension ${ }^{12}$; and meta-inflammation, collectively known as the metabolic syndrome. This predisposes multiple degenerative conditions of ageing ${ }^{13}$. Lifestyle modification

This article is protected by copyright. All rights reserved. 
including weight loss and exercise is the mainstay of treatment of the metabolic syndrome ${ }^{14}$. Despite this, there have been a number of barriers to physician recommendation of these measures in older people with obesity. These include the observation that obesity can be associated with lower cardiovascular and all-cause mortality in older people (the "obesity paradox $" 15$ ), fear regarding lean mass $\operatorname{loss}^{16}$, and a lack of evidence to support the safety and efficacy of weight loss measures in the older population. However, it has recently been demonstrated that weight loss and exercise can improve cardiovascular and diabetes markers in older adults with obesity ${ }^{6,7}$. This study demonstrates that weight loss achieved with a 12 -week period of VLCD plus exercise can improve glycaemic, cholesterol and blood pressure parameters in older adults with obesity.

In this study, which included participants with and without type-2 diabetes, there was a significant lowering in $\mathrm{HbA}_{1 \mathrm{C}}$ in $\mathrm{Ex} / \mathrm{VLCD}$ over the 12-week study period, of $0.59 \%$. For comparison, in people with diabetes, the $\mathrm{HbA}_{1 \mathrm{C}}$ lowering is $1.12 \%$ with Metformin monotherapy ${ }^{17}$. Weight loss induced by VLCDs has previously been demonstrated to restore normoglycaemia in persons with diabetes ${ }^{18}$, and these changes are often durable if the lower weight is maintained ${ }^{19}$.

Currently, it is unclear as to how to manage hypoglycaemic medication in people with diabetes undergoing a VLCD - previous published recommendations recommended halving insulin and halving or ceasing sulphonylureas ${ }^{10}$. However, this approach led to hypoglycaemia in this study. In another study ${ }^{18}$, diabetes medications were ceased altogether. From a practical standpoint, 
changes to diabetes medicines in those losing weight on a low carbohydrate diet will need to be individualised, depending on medication, baseline glycaemic control and diabetic complications, adherence to the VLCD, and the intended $\mathrm{HbA}_{1 \mathrm{c}}$ target for the individual.

The pathophysiology of hypertension in obesity is complex. Insulin resistance and hyperuricemia contribute to increased renin-angiotensin system activation and increased sympathetic outflow (which is also contributed to by obstructive sleep apnoea). A chronic inflammatory state causes vascular endothelial dysfunction, renal changes favouring sodium retention, and myocardial dysfunction ${ }^{20}$. Weight loss can decrease insulin resistance and inflammatory burden, and improve obstructive sleep apnoea ${ }^{3,21}$. Those in Ex/VLCD had significant decreases in systolic blood pressure in keeping with their significant weight loss. This necessitated reduction in antihypertensive medication in many of these participants, to avoid symptomatic hypotension, which is a major cause of morbidity in older adults. A large trial of $8 \mathrm{mg}$ perindopril in secondary prevention of cardiovascular disease demonstrated a $9 / 4 \mathrm{mmHg}$ lowering of blood pressure after a 4-6 week run in period ${ }^{22}$. The ideal blood pressure target for older adults is the topic of some debate. The SPRINT study ${ }^{23}$ demonstrated that cardiovascular outcomes are improved when blood pressure is controlled to a target of $120 \mathrm{mmHg}$ rather than $140 \mathrm{mmHg}$ in people over the age of 75, with a trend toward higher adverse events such as orthostatic hypotension and acute kidney injury in the intensively treated group. This study demonstrated a significant blood pressure lowering despite withdrawal of antihypertensive agents, though, on average, not to $120 \mathrm{mmHg}$ systolic. 
There was a significant reduction in triglycerides in Ex/VLCD, The triglyceride lowering effect was lower than, but comparable to, long courses of fibrate medication ${ }^{24}$. Previous studies of weight loss in older adults have shown similar results ${ }^{7}$. Triglycerides are an emerging treatment target as their level is proportional to risk of cardiovascular disease ${ }^{25}$, and triglyceride levels correlate with cerebral amyloid burden ${ }^{26}$. However, it is currently unclear whether triglyceride lowering with medications (or indeed diet) reduce the risk of cardiovascular disease in the long $\operatorname{term}^{25,27}$.

Low-grade, systemic inflammation (meta-inflammation) is central to the pathophysiology of cardiovascular disease $^{28}$ and degenerative diseases of ageing ${ }^{1}$. Reduction in markers of inflammation such as IL-6, TNF- \pm and CRP have been observed in older adults undergoing substantial weight loss, proportional to body fat $\operatorname{lost}^{29-31}$. In this study, no significant reductions in any of the markers were observed. A potential reason for the discrepancy is that these previous studies are longer term; 18 months compared with the 12 weeks of this study.

Obtaining tighter blood pressure and glycaemic control can be risky in older people, as it can predispose to polypharmacy and adverse drug reactions $\mathrm{s}^{32}$. Cardiovascular and hypoglycaemic medication-related adverse events are common causes of hospitalisation in older adults ${ }^{33}$. This study demonstrated that, at least in the short term, improvements in cardiovascular and glycaemic markers similar to that achieved with common medications could be obtained with substantial weight loss. However, this study also illustrated the need for close observation of 
blood pressure and glucose in older persons losing weight with a VLCD, as reduction in these parameters was rapid.

Limitations to this study include relatively low numbers, and a heterogeneous study population (ie people with and without diabetes). While the study demonstrated improvements in the short term, it would be important to elucidate whether these findings could be maintained in the long term. The gold standard for measuring blood pressure is with 24 hour monitoring; this was not performed in this study but would be important to include in future studies in this age group. This study adds to the expanding pool of data regarding management of older adults with obesity, particularly with regards to the use of VLCDs. The study demonstrated rapid improvements in systolic blood pressure, glycaemic measures and triglycerides on those participants randomised to a VLCD. The significance of this finding is that, if an older adult with obesity presents with poorly controlled type 2 diabetes, hypertension or triglycerides despite medication, weight loss with a VLCD may be a reasonable first step before adding further medication.

In older adults with obesity, weight loss has pleiotrophic positive effects on cardiovascular risk markers, and these were marked in those who underwent a short treatment regimen with exercise and a VLCD. Weight loss is an underutilized preventative measure for cardiovascular disease in this group, especially given the potential for reduction in polypharmacy.

Conflicts of interest: None of the authors declare any conflict of interest from this work. 


\section{Acknowledgements / Author contributions:}

$\mathrm{CH}$, WKL and JP designed the study. $\mathrm{CH}$ collected the data. $\mathrm{CH}$ and LAP undertook the statistical analysis. ML and RL selected the appropriate tests for inflammatory markers, carried out the tests, and contributed to the manuscript on this topic. $\mathrm{CH}$ wrote the initial draft of the manuscript and sought the input of all of the other authors for the final manuscript submission. The research project was funded a National Health and Medical Research Council Project grant (APP1044334), and CH was supported by a National Health and Medical Research Council Medical/Dental Postgraduate Scholarship (APP1056512).

Conflict of interest statement: None related to this study. $\mathrm{CH}$ and JP have received lecture fees from Novo Nordisk and iNova Pharmaceuticals. JP is on the medical advisory board for

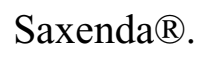

\section{REFERENCES:}

1. Cevenini E, Monti D, Franceschi C. Inflamm-ageing. Current Opinion In Clinical Nutrition And Metabolic Care. 2013;16(1): 14-20.

2. Ferrucci L, Fabbri E. Inflammageing: chronic inflammation in ageing, cardiovascular disease, and frailty. Nature Reviews. Cardiology. 2018;15(9): 505-522.

3. Anderson JW, Brinkman-Kaplan VL, Lee H, Wood CL. Relationship of weight loss to cardiovascular risk factors in morbidly obese individuals. Journal Of The American College Of Nutrition. 1994;13(3): 256-261.

This article is protected by copyright. All rights reserved. 
4. Bazzano LA, Hu T, Reynolds K, et al. Effects of low-carbohydrate and low-fat diets: a randomized trial. Annals Of Internal Medicine. 2014;161(5): 309-318.

5. Gibbs BB, Brancati FL, Chen H, et al. Effect of improved fitness beyond weight loss on cardiovascular risk factors in individuals with type 2 diabetes in the Look AHEAD study. European Journal Of Preventive Cardiology. 2014;21(5): 608-617.

6. Villareal DT, Banks MR, Patterson BW, Polonsky KS, Klein S. Weight loss therapy improves pancreatic endocrine function in obese older adults. Obesity (Silver Spring, Md.). 2008;16(6): 1349-1354.

7. Villareal DT, Miller BV, 3rd, Banks M, Fontana L, Sinacore DR, Klein S. Effect of lifestyle intervention on metabolic coronary heart disease risk factors in obese older adults. The American Journal Of Clinical Nutrition. 2006;84(6): 1317-1323.

8. Messier SP, Mihalko SL, Legault C, et al. Effects of intensive diet and exercise on knee joint loads, inflammation, and clinical outcomes among overweight and obese adults with knee osteoarthritis: the IDEA randomized clinical trial. JAMA. 2013;310(12): 1263-1273.

9. Haywood CJ, Prendergast LA, Purcell K, et al. Very Low Calorie Diets for Weight Loss in Obese Older Adults-A Randomized Trial. The Journals Of Gerontology. Series A, Biological Sciences And Medical Sciences. 2017.

10. Baker S, Jerums G, Proietto J. Effects and clinical potential of very-low-calorie diets (VLCDs) in type 2 diabetes. Diabetes Res Clin Pract. 2009;85(3): 235-242.

This article is protected by copyright. All rights reserved. 
11. Broughton DL, James OW, Alberti KG, Taylor R. Peripheral and hepatic insulin sensitivity in healthy elderly human subjects. Eur J Clin Invest. 1991;21(1): 13-21.

12. Sun Z. Aging, arterial stiffness, and hypertension. Hypertension (Dallas, Tex.: 1979). 2015;65(2): 252-256.

13. Pawelec G, Goldeck D, Derhovanessian E. Inflammation, ageing and chronic disease. Current Opinion In Immunology. 2014;29: 23-28.

14. Grundy SM, Cleeman JI, Daniels SR, et al. Diagnosis and management of the metabolic syndrome: an American Heart Association/National Heart, Lung, and Blood Institute scientific statement. Current Opinion In Cardiology. 2006;21(1): 1-6.

15. Lavie CJ, McAuley PA, Church TS, Milani RV, Blair SN. Obesity and cardiovascular diseases: implications regarding fitness, fatness, and severity in the obesity paradox. Journal Of The American College Of Cardiology. 2014;63(14): 1345-1354.

16. Miller SL, Wolfe RR. The danger of weight loss in the elderly. The Journal Of Nutrition, Health \& Aging. 2008;12(7): 487-491.

17. Hirst JA, Farmer AJ, Ali R, Roberts NW, Stevens RJ. Quantifying the effect of metformin treatment and dose on glycemic control. Diabetes Care. 2012;35(2): 446-454.

18. Steven S, Taylor R. Restoring normoglycaemia by use of a very low calorie diet in longand short-duration Type 2 diabetes. Diabetic Medicine: A Journal Of The British Diabetic Association. 2015;32(9): 1149-1155.

This article is protected by copyright. All rights reserved. 
19. Steven S, Hollingsworth KG, Al-Mrabeh A, et al. Very Low-Calorie Diet and 6 Months of Weight Stability in Type 2 Diabetes: Pathophysiological Changes in Responders and Nonresponders. Diabetes Care. 2016;39(5): 808-815.

20. DeMarco VG, Aroor AR, Sowers JR. The pathophysiology of hypertension in patients with obesity. Nature Reviews. Endocrinology. 2014;10(6): 364-376.

21. Chirinos JA, Gurubhagavatula I, Teff K, et al. CPAP, weight loss, or both for obstructive sleep apnea. The New England Journal Of Medicine. 2014;370(24): 2265-2275.

22. Fox KM. Efficacy of perindopril in reduction of cardiovascular events among patients with stable coronary artery disease: randomised, double-blind, placebo-controlled, multicentre trial (the EUROPA study). Lancet. 2003;362(9386): 782-788.

23. Williamson JD, Supiano MA, Applegate WB, et al. Intensive vs Standard Blood Pressure Control and Cardiovascular Disease Outcomes in Adults Aged e 75 Years: A Randomized Clinical Trial. JAMA. 2016;315(24): 2673-2682.

24. Fruchart JC, Brewer HB, Jr., Leitersdorf E. Consensus for the use of fibrates in the treatment of dyslipoproteinemia and coronary heart disease. Fibrate Consensus Group. The American Journal Of Cardiology. 1998;81(7): 912-917.

25. Maki KC, Dicklin MR. Do triglyceride-lowering drugs decrease risk of cardiovascular disease? Current Opinion In Lipidology. 2017.

26. Choi HJ, Byun MS, Yi D, et al. Association Between Serum Triglycerides and Cerebral Amyloidosis in Cognitively Normal Elderly. The American Journal Of Geriatric

This article is protected by copyright. All rights reserved. 
Psychiatry: Official Journal Of The American Association For Geriatric Psychiatry. 2016;24(8): 604-612.

27. Nordestgaard BG, Varbo A. Triglycerides and cardiovascular disease. Lancet (London, England). 2014;384(9943): 626-635.

28. Libby P, Theroux P. Pathophysiology of coronary artery disease. Circulation. 2005;111(25): 3481-3488.

29. Beavers KM, Beavers DP, Newman JJ, et al. Effects of total and regional fat loss on plasma CRP and IL-6 in overweight and obese, older adults with knee osteoarthritis. Osteoarthritis And Cartilage. 2015;23(2): 249-256.

30. Beavers KM, Ambrosius WT, Nicklas BJ, Rejeski WJ. Independent and combined effects of physical activity and weight loss on inflammatory biomarkers in overweight and obese older adults. Journal Of The American Geriatrics Society. 2013;61(7): 1089-1094.

31. Nicklas BJ, Ambrosius W, Messier SP, et al. Diet-induced weight loss, exercise, and chronic inflammation in older, obese adults: a randomized controlled clinical trial. The American Journal Of Clinical Nutrition. 2004;79(4): 544-551.

32. Munger MA. Polypharmacy and combination therapy in the management of hypertension in elderly patients with co-morbid diabetes mellitus. Drugs \& Aging. 2010;27(11): 871883.

This article is protected by copyright. All rights reserved. 
33. Budnitz DS, Lovegrove MC, Shehab N, Richards CL. Emergency hospitalizations for adverse drug events in older Americans. The New England Journal Of Medicine. 2011;365(21): 2002-2012.

This article is protected by copyright. All rights reserved. 


\section{TABLES}

Table 1 - Demographics

\begin{tabular}{|l|c|c|c|c|}
\hline Marker & Ex/HE $(n=36)$ & Ex/Diet $(n=40)$ & Ex/VLCD $(n=41)$ & P \\
\hline Number of Females & 23 & 24 & 25 & 0.937 \\
\hline Hypertension & 33 & 37 & 39 & 0.818 \\
\hline Dyslipidemia & 23 & 32 & 37 & 0.236 \\
\hline Ischaemic Heart Disease & 4 & 12 & 8 & 0.123 \\
\hline Obstructive Sleep Apnoea & 12 & 12 & 13 & 0.952 \\
\hline Type 2 Diabetes & 10 & 13 & 14 & 0.826 \\
\hline Impaired Fasting Glucose & 15 & 11 & 10 & 0.224 \\
\hline Metabolic Syndrome* & 29 & 35 & 33 & 0.636 \\
\hline
\end{tabular}

$\mathrm{N}=117,72$ females, 45 males.

Mean age 70.2 years (range 65-85).

Mean weight at randomisation $105.7 \mathrm{~kg}$ (76.7-146.1)

Mean BMI $40.1 \mathrm{~kg} / \mathrm{m}^{2}$ (range 32.34-57.07)

${ }^{*}$ Metabolic Syndrome as defined by International Diabetes Federation Definition

${ }^{\S}$ Pearson Chi-Square Test 
Baseline and change values are stated as Mean (Standard Error of the Mean), changes are absolute (not percentage) values

* denotes changes that are statistically significant $(P<0.05)$

$\S$ One-Way P, Kruskal-Wallis test

$\sim$ Unshaded boxes: absolute mean ( $95 \%$ confidence interval) difference between groups, shaded boxes: percentage differences (95\% confidence interval) between groups - See statistical methods section.

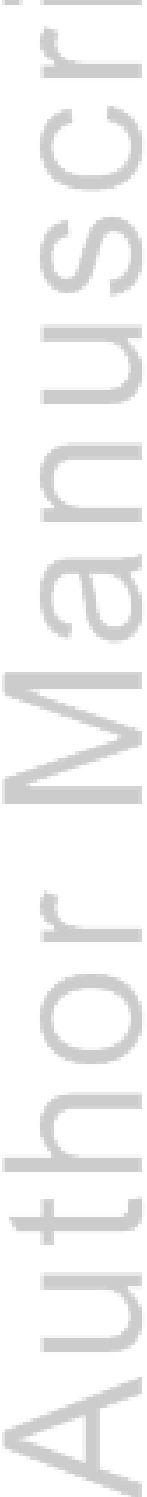

This article is protected by copyright. All rights reserved. 
Table 3 - Changes in Medications

\begin{tabular}{|l|l|l|l|l|}
\hline Change & Ex/HE & Ex/Diet & Ex/VLCD & $\mathrm{P}^{*}$ \\
\hline $\begin{array}{l}\text { Any Medication Change } \\
\mathrm{N}=102 \text { completers }\end{array}$ & $4 / 29$ & $7 / 36$ & $16 / 37$ & 0.017 \\
\hline $\begin{array}{l}\text { Change in Antihypertensive medication } \\
\mathrm{N}=95 \text { completers with hypertension }\end{array}$ & $0 / 27$ & $5 / 32$ & $11 / 36$ & 0.003 \\
\hline $\begin{array}{l}\text { Change in Diabetes Medication } \\
\mathrm{N}=35 \text { completers with type 2 diabetes }\end{array}$ & $2 / 9$ & $3 / 12$ & $10 / 14$ & 0.028 \\
\hline
\end{tabular}

*Fisher's exact 


\section{Please wait...}

If this message is not eventually replaced by the proper contents of the document, your PDF viewer may not be able to display this type of document.

You can upgrade to the latest version of Adobe Reader for Windows®, Mac, or Linux® by visiting http://www.adobe.com/go/reader_download.

For more assistance with Adobe Reader visit http://www.adobe.com/go/acrreader.

Windows is either a registered trademark or a trademark of Microsoft Corporation in the United States and/or other countries. Mac is a trademark
of Apple Inc., registered in the United States and other countries. Linux is the registered trademark of Linus Torvalds in the U.S. and other countries. 


\section{Please wait...}

If this message is not eventually replaced by the proper contents of the document, your PDF viewer may not be able to display this type of document.

You can upgrade to the latest version of Adobe Reader for Windows®, Mac, or Linux® by visiting http://www.adobe.com/go/reader_download.

For more assistance with Adobe Reader visit http://www.adobe.com/go/acrreader.

Windows is either a registered trademark or a trademark of Microsoft Corporation in the United States and/or other countries. Mac is a trademark
of Apple Inc., registered in the United States and other countries. Linux is the registered trademark of Linus Torvalds in the U.S. and other countries. 


\section{Please wait...}

If this message is not eventually replaced by the proper contents of the document, your PDF viewer may not be able to display this type of document.

You can upgrade to the latest version of Adobe Reader for Windows®, Mac, or Linux® by visiting http://www.adobe.com/go/reader_download.

For more assistance with Adobe Reader visit http://www.adobe.com/go/acrreader.

Windows is either a registered trademark or a trademark of Microsoft Corporation in the United States and/or other countries. Mac is a trademark
of Apple Inc., registered in the United States and other countries. Linux is the registered trademark of Linus Torvalds in the U.S. and other countries. 


\section{Please wait...}

If this message is not eventually replaced by the proper contents of the document, your PDF viewer may not be able to display this type of document.

You can upgrade to the latest version of Adobe Reader for Windows®, Mac, or Linux® by visiting http://www.adobe.com/go/reader_download.

For more assistance with Adobe Reader visit http://www.adobe.com/go/acrreader.

Windows is either a registered trademark or a trademark of Microsoft Corporation in the United States and/or other countries. Mac is a trademark
of Apple Inc., registered in the United States and other countries. Linux is the registered trademark of Linus Torvalds in the U.S. and other countries. 


\section{Please wait...}

If this message is not eventually replaced by the proper contents of the document, your PDF viewer may not be able to display this type of document.

You can upgrade to the latest version of Adobe Reader for Windows ${ }^{\circledR}$, Mac, or Linux® by visiting http://www.adobe.com/products/acrobat/readstep2.html.

For more assistance with Adobe Reader visit http://www.adobe.com/support/products/ acrreader.html.

Windows is either a registered trademark or a trademark of Microsoft Corporation in the United States and/or other countries. Mac is a trademark of Apple Inc., registered in the United States and other countries. Linux is the registered trademark of Linus Torvalds in the U.S. and other countries. 


\section{Please wait...}

If this message is not eventually replaced by the proper contents of the document, your PDF viewer may not be able to display this type of document.

You can upgrade to the latest version of Adobe Reader for Windows®, Mac, or Linux® by visiting http://www.adobe.com/go/reader_download.

For more assistance with Adobe Reader visit http://www.adobe.com/go/acrreader.

Windows is either a registered trademark or a trademark of Microsoft Corporation in the United States and/or other countries. Mac is a trademark
of Apple Inc., registered in the United States and other countries. Linux is the registered trademark of Linus Torvalds in the U.S. and other countries. 


\section{Obesity in older adults: effect of degree of weight loss on cardiovascular markers and medications.}

Cilla J Haywood ${ }^{1,3,4}$

Luke A Prendergast ${ }^{6}$

Ratana $\operatorname{Lim}^{7}$

Martha Lappas ${ }^{7}$

Wen Kwang Lim ${ }^{2,5}$

Joseph Proietto ${ }^{1,4}$

1. Department of Medicine (Austin Health), University of Melbourne, Heidelberg Heights, Australia

2. Department of Medicine (Royal Melbourne Hospital), University of Melbourne, Parkville, Australia

3. Department of Aged Care, Austin Health, Heidelberg, Australia

4. Department of Endocrinology, Austin Health, Heidelberg, Australia

5. Department of Aged Care, Royal Melbourne Hospital, Parkville, Australia

6. Mathematics and Statistics, LaTrobe University, Bundoora, Australia

7. Obstetrics, Nutrition and Endocrinology Group, Department of Obstetrics and Gynaecology, University of Melbourne, Mercy Hospital for Women, Heidelberg, Victoria, Australia

Corresponding Author: Dr. Cilla Haywood

chaywood@unimelb.edu.au

Metabolic Disorders Centre

$1^{\text {st }}$ Floor, Centaur Wing, Heidelberg Repatriation Hospital

Waterdale Rd, Heidelberg Heights, Victoria 3081, Australia

$\mathrm{Ph}+61407340863$

Orcid ID for Cilla Haywood: https://orcid.org/0000-0002-8127-7014

Running title: Weight loss in older persons with obesity

Keywords: Obesity, older adults, hypertension, very low calorie diet

Conflict of Interest Statement: The authors report no conflicts of interest directly related to this study. Related to broader study of obesity, $\mathrm{CH}$ and JP have received lecture fees from Novo Nordisk and iNova Pharmaceuticals (Duromine ${ }^{\circledR}$ ). JP is on the medical advisory board for Saxenda ${ }^{\circledR}$ (Novo Nordisk Pharmaceuticals)

Acknowledgements: The research project was funded a National Health and Medical Research Council Project grant (APP1044334), and CH was supported by a National Health and Medical Research Council Medical/Dental Postgraduate Scholarship (APP1056512). 


\section{University Library}

\section{- $\mathrm{M}$ IIN E R VA A gateway to Melbourne's research publications}

Minerva Access is the Institutional Repository of The University of Melbourne

Author/s:

Haywood, CJ;Prendergast, LA;Lim, R;Lappas, M;Lim, WK;Proietto, J

Title:

Obesity in older adults: Effect of degree of weight loss on cardiovascular markers and medications

Date:

2019-08-01

Citation:

Haywood, C. J., Prendergast, L. A., Lim, R., Lappas, M., Lim, W. K. \& Proietto, J. (2019). Obesity in older adults: Effect of degree of weight loss on cardiovascular markers and medications. CLINICAL OBESITY, 9 (4), https://doi.org/10.1111/cob.12316.

Persistent Link:

http://hdl.handle.net/11343/286001 